\title{
Decline in Prescription Opioids Attributable to Decreases in Long-Term Use: A Retrospective Study in the Veterans Health Administration 2010-2016
}

\author{
Katherine Hadlandsmyth, $P h D^{1,2}$, Hilary Mosher, $M D^{1,3}$, Mark W. Vander Weg, $P h D^{1,3}$, and \\ Brian C. Lund, PharmD ${ }^{7}$ \\ 'Center for Comprehensive Access and Delivery Research and Evaluation (CADRE), lowa City VA Healthcare System, lowa City, IA, USA; \\ ${ }^{2}$ Department of Anesthesia, Carver College of Medicine, University of lowa, lowa City, IA, USA; ${ }^{3}$ Department of Internal Medicine, Carver College \\ of Medicine, University of lowa, lowa City, IA, USA.
}

BACKGROUND: Improved understanding of temporal trends in short- and long-term opioid prescribing may inform efforts to curb the opioid epidemic.

OBJECTIVE: To characterize the prevalence of short- and long-term opioid prescribing in the Veterans Health Administration (VHA) from 2010 to 2016.

DESIGN: Observational cohort study using VHA databases.

PARTICIPANTS: All patients receiving at least one outpatient prescription through the VHA during calendar years 2010 through 2016.

MAIN MEASURES: Prevalence of opioid use from 2010 through 2016, stratified by short-term, intermediateterm, and long-term use. Temporal trends in discontinuation among existing long-term users and initiation of new long-term use and the net impact on rates of longterm opioid use. Relative likelihood of transitioning to long-term opioid use contrasted with use patterns in the prior year.

KEY RESULTS: The prevalence of opioid prescribing was $20.8 \%$ in 2010 , peaked at $21.2 \%$ in 2012 , and declined annually to $16.1 \%$ in 2016. Between 2010 and 2016, reductions in long-term opioid prescribing accounted for $83 \%$ of the overall decline in opioid prescription fills. Comparing data from 2010-2011 to data from 2015-2016, declining rates in new long-term use accounted for more than $90 \%$ of the decreasing prevalence of long-term opioid use in the VHA, whereas increases in cessation among existing long-term users accounted for less than $10 \%$. The relative risk of transitioning to long-term use during 2016 was 6.5 (95\% CI: 6.4, 6.7) among short-term users and 35.5 (95\% CI: 34.8, 36.3) among intermediate users, relative to patients with no opioid prescriptions filled during 2015.

CONCLUSIONS: Opioid prescribing trends followed similar trajectories in VHA and non-VHA settings, peaking around 2012 and subsequently declining. However, changes in long-term opioid prescribing accounted for most of the decline in the VHA. Recent VA opioid initiatives may be preventing patients from initiating long-term use. This may offer valuable lessons generalizable to other healthcare systems.

Received August 30, 2017

Revised November 16, 2017

Accepted December 14, 2017

Published online January 29, 2018
KEY WORDS: opioid; veteran; prevalence.

J Gen Intern Med 33(6):818-24

DOI: $10.1007 / \mathrm{s} 11606-017-4283-8$

(C) Society of General Internal Medicine (outside the USA) 2018

\section{INTRODUCTION}

Rapid and widespread escalation in long-term opioid prescribing over recent decades have revealed a spectrum of harms including opioid use disorder, transition to heroin use, bowel dysfunction, endocrine dysfunction, excess sedation, vehicular accidents, respiratory depression, and fatal overdoses. ${ }^{1-7}$ Growing awareness has led prescribers, policymakers, and the lay community to enact numerous efforts to "turn the tide" on the opioid epidemic, including prescription drug monitoring programs, ${ }^{1,2}$ hydrocodone rescheduling, ${ }^{3}$ state-based limits on prescribing, ${ }^{4}$ the 2016 Centers for Disease Control and Prevention (CDC) guidelines on opioids in chronic pain management, ${ }^{5}$ the Comprehensive Addiction and Recovery Act, ${ }^{6}$ and an array of local awareness campaigns.

A recent study using data from nearly $90 \%$ of US pharmacies reported a key reversal in per capita opioid prescribing, shifting to a downward trend in $2012 .^{7}$ Despite this encouraging endorsement of efforts to curtail use and availability, opioid prescribing remained approximately three times as high in 2015 as in 1999. Moreover, decreases were limited to opioid prescriptions of $<30$ days' supply, whereas prescriptions for $\geq 30$ days' supply continued increasing through 2015 . In composite, the average days' supply per prescription rose from 13.3 in 2006 to 17.7 in 2015 . These patterns suggest that recent decreases in opioid prescribing were attributable to decreases in incident prescribing, largely driven by treatment of acute pain. In contrast, expansion in prescriptions of $\geq 30$ days' supply suggests that long-term opioid use, likely for chronic pain, has persisted and perhaps even increased in recent years. ${ }^{\text {? }}$

Secular trends in opioid prescribing have also impacted the Veteran Health Administration (VHA), the largest nationally integrated health system in the US. From 2004 to 2012, the prevalence of opioid prescribing nearly doubled. ${ }^{8}$ Since this time, VHA has employed several strategies to combat the 
opioid epidemic, including deployment of educational initiatives, improving pain management programs, creating risk mitigation systems to enhance opioid safety, and expanding access to addiction treatment. ${ }^{9}$ Mirroring changes in the private sector, escalating opioid prevalence in the VHA slowed and reversed toward a downward trend over the period from 2012 to $2014 .{ }^{9}$ However, VHA prescribing trends have not been explicitly segregated into trends in short-term versus long-term use. This distinction is critical in understanding the impact of VHA's strategy to combat the opioid epidemic.

Therefore, the primary objective of this study was to characterize the overall prevalence of opioid prescribing in the VHA from 2010 through 2016, stratified according to duration of use categories: short-term, intermediate-term, and long-term. In addition to prevalence, opioid prescribing trends were expressed in terms of number of prescription fills and days' supply dispensed. These alternative metrics provided complementary data concerning patterns of opioid prescribing and allowed examination of trends in days' supply per prescription for comparison to prior evaluation in the non-VHA setting. ${ }^{7}$ Given the key role of long-term opioid use, we further examined changes within this patient group, specifically charting the number of patients entering as new long-term users and exiting through cessation of long-term use, and how these prescribing patterns changed over time. Finally, we contrasted no use, short-term use, and intermediate-term opioid use groups and their relative likelihood of transitioning to long-term use in the subsequent year, as well as changes in the likelihood of transitioning to long-term use over time.

\section{METHODS}

\section{Data Sources and Patients}

National administrative data from the VA Corporate Data Warehouse were accessed using the VA Informatics and Computing Infrastructure. Prescriptions dispensed during calendar years 2010 through 2016 were identified using outpatient pharmacy data sets. Annual cohorts of VHA pharmacy users were constructed and defined by the presence of at least one outpatient prescription dispensed during that calendar year. This study was approved by the University of Iowa Institutional Review Board and the Iowa City Veterans Administration Research and Development Committee.

\section{Opioid Prescribing Measures}

Opioid medications considered were non-injectable dosage forms of butorphanol, fentanyl, hydrocodone, hydromorphone, levorphanol, meperidine, methadone, morphine, oxycodone, oxymorphone, pentazocine, and tapentadol dispensed as outpatient prescriptions. The prevalence of opioid use for each year was determined by the number of patients dispensed at least one opioid prescription divided by the number of pharmacy users in that year. Additional measures of opioid prescribing included total opioid prescriptions dispensed, supply days dispensed, milligrams of morphine equivalents dispensed, ${ }^{10}$ and mean supply days per fill. Estimated daily doses were calculated for each prescription based on unit strength, quantity, and days' supply values, and were used to assess a time-weighted mean daily dose for each patient over the course of a calendar year, expressed in milligrams of morphine equivalents per day.

Duration of opioid use was defined at the patient level and determined for each calendar year as one of four categories: no use, short-term (acute) use, intermediate use, or long-term (chronic) use. Patients with no opioid prescriptions filled during the calendar year were classified as no use. Patients with either a single opioid prescription or two prescriptions separated by more than 90 days were classified as short-term use. This second criterion was used to allow patients to have up to two discrete episodes of opioid therapy during a given year without being placed into a category of higher opioid use. Long-term use was defined as a period of $>90$ days of continuous opioid use within the calendar year, as established by the cabinet supply approach. ${ }^{11}$ Patients with any other pattern of opioid use were classified as intermediate use. The mean supply days dispensed during 2016 were 15.0, 58.8, and 305.8 days for short-term, intermediate, and long-term opioid use groups, respectively.

\section{Analysis}

Descriptive statistics were used to quantify trends in opioid prescribing metrics over time in aggregate and separately for short-term, intermediate, and long-term use categories. Given the importance of long-term opioid use, we further examined changes within this patient group by charting the number of patients entering as new long-term users and exiting through cessation of long-term use. We specifically examined these transitions in long-term opioid use from 2010 to 2011 and again from 2015 to 2016 to determine how these prescribing patterns changed over time. Log-binomial regression was used to determine the relative risk of becoming a new long-term user based on duration of use category from the prior year (no use, short-term use, or intermediate use).

\section{RESULTS}

The number of veterans dispensed a VHA prescription rose from 4,635,313 in 2010 to 4,991,926 in 2016. The prevalence of opioid prescribing was $20.8 \%(962,193 / 4,635,313)$ in 2010 , peaked at $21.2 \%(1,017,826 / 4,793,253)$ in 2012 , and declined annually to $16.1 \%(803,888 / 4,991,926)$ in 2016 (Fig. 1). The prevalence of long-term and intermediate use decreased by approximately one-third: long-term use declined from 9.5\% $(453,100 / 4,793,253$ in 2012) to $6.2 \%(309,881 / 4,991,926$ in 2016) and intermediate use declined from $4.4 \%$ (210,555/ $4,793,253$ in 2012) to $3.0 \%(150,884 / 4,991,926$ in 2016). In 


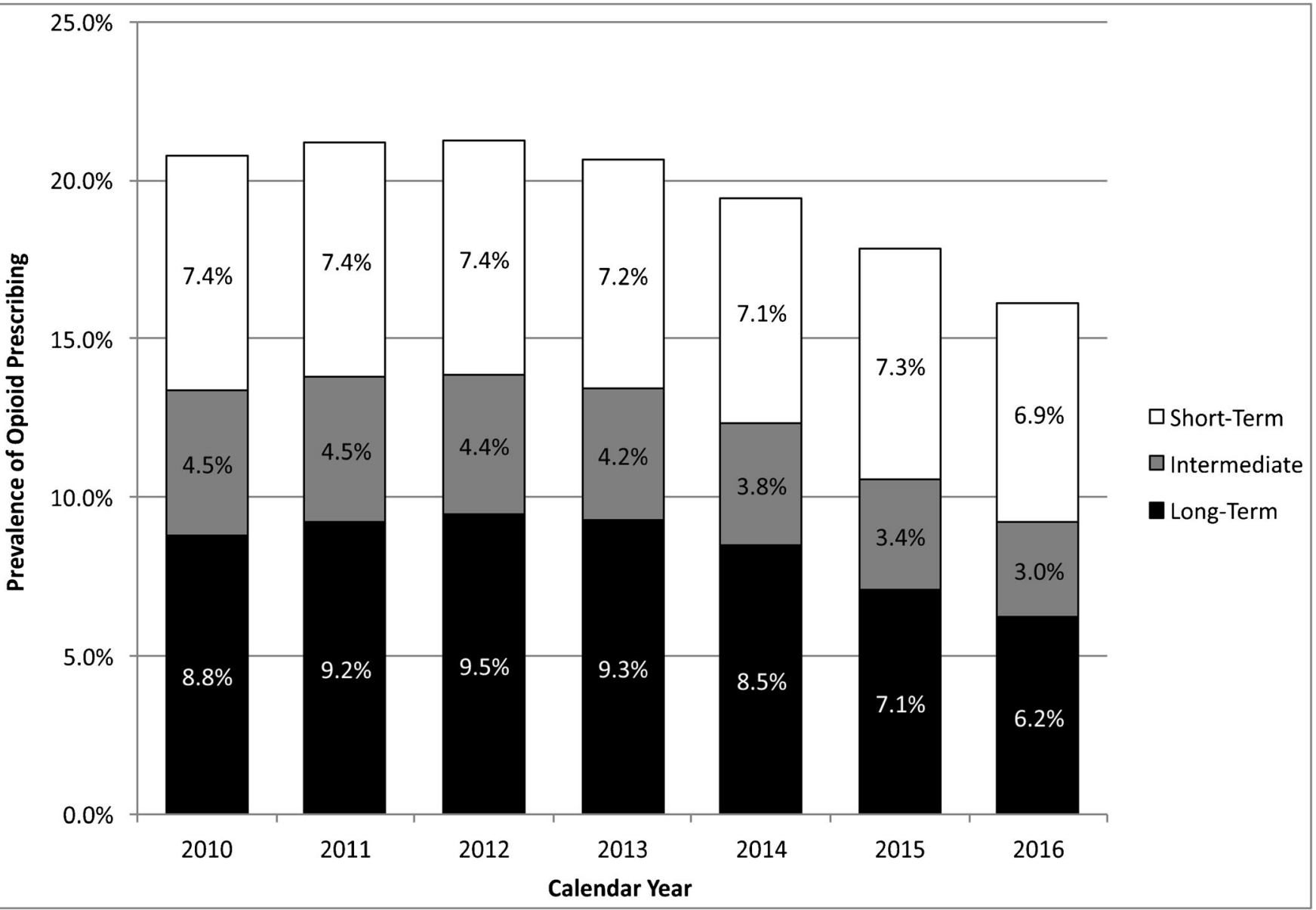

Figure 1 The changing prevalence of opioid prescribing in the VA healthcare system.

contrast, short-term opioid use remained relatively constant throughout the observation period, ranging from 343,556 $(7.4 \%)$ in 2010 to $354,171(7.4 \%)$ in 2012 and 343,123 $(6.9 \%)$ in 2016.

Supplemental measures of opioid prescribing paralleled the trend in decreasing prevalence. The total number of opioid prescription fills declined by $24.4 \%$, supply days by $27.2 \%$, and morphine equivalents by $32.2 \%$ from 2010 to 2016 (Table 1). The reduction in opioid prescribing was unevenly distributed across duration of use categories (Table 1). Total opioid prescriptions decreased by $25.2 \%$ among long-term users and $31.8 \%$ among intermediate-term users, but only $1.6 \%$ among short-term users. In total, changes in prescribing among long-term opioid users accounted for $83.0 \%(1,125,237 / 1,355,217)$ of the overall decline in opioid prescription fills in the VHA during the observation period, $81.9 \%(33,344,343 / 40,692,111)$ of the change in supply days dispensed, and $90.5 \%(1785 \mathrm{M} / 1973 \mathrm{M})$ of the change in morphine equivalents. The number of supply days per prescription was relatively stable among long-term users $(1.7 \%$ decrease), but declined $15.0 \%$ (3.2 days) among intermediate users and $16.9 \%$ (2.7 days) among short-term users. Estimated daily opioid doses were substantially higher among long-term users, $47.0 \mathrm{mg}$ of morphine equivalents in 2016, compared to
$27.0 \mathrm{mg}$ and $24.7 \mathrm{mg}$ among intermediate and short-term users, respectively. Daily doses declined slightly over time among long-term users, down from $50.2 \mathrm{mg}$ in 2010.

Given the declining prevalence of long-term opioid use, we sought to determine the extent to which this was explained by an increase in cessation among existing long-term users versus a reduction in patients becoming new long-term users (Fig. 2). Therefore, we examined patterns of transitioning into and out of long-term use over subsequent years at two time intervals: 2010 to 2011 and 2015 to 2016 . Of 4,125,454 patients who were VHA pharmacy users during 2010 and 2011, 389,890 $(9.5 \%)$ were long-term opioid users in 2010 . The majority $(82.5 \% ; n=321,710)$ persisted as long-term users in the subsequent year, while the remaining $17.5 \%(n=68,180)$ of patients did not persist with long-term use in 2011. However, 103,793 patients became new long-term users during 2011, resulting in a net increase of 35,613 long-term opioid users from 2010 to 2011. Applying the same approach to the later period of 2015-2016 revealed some important differences. Notably, 73,052 former long-term users discontinued, but only 44,359 new long-term users were observed, resulting in a net reduction of 28,693 long-term users from 2015 to 2016 . Given the net decrease in the number of new long-term users over time $(103,793-44,359=59,434)$ and net increase in patients 
Table 1 Distribution of Opioid Prescribing Metrics by Duration of Opioid Use Category

\begin{tabular}{|c|c|c|c|}
\hline Opioid Prescribing Metrics & 2010 & 2016 & Reduction \\
\hline Prescription Fills & Count (column \%) & Count (column \%) & Count (\% reduction) \\
\hline Total & $5,550,308(100)$ & 4,195,091 (100) & $1,355,217(24.4)$ \\
\hline Long-term use & $4,470,200(80.5)$ & $3,344,963(79.7)$ & $1,125,237(25.2)$ \\
\hline Intermediate use & $696,909(12.6)$ & $475,039(11.3)$ & $221,870(31.8)$ \\
\hline Short-term use & $382,199(6.9)$ & $375,899(9.0)$ & $6300(1.6)$ \\
\hline Supply Days Dispensed & Count (column \%) & Count (column \%) & Count (\% reduction) \\
\hline Total & $149,457,582(100)$ & $108,765,471(100)$ & $40,692,111(27.2)$ \\
\hline Long-term use & $128,105,586(85.7)$ & $94,761,243(87.1)$ & $33,344,343(26.0)$ \\
\hline Intermediate use & $15,014,817(10.0)$ & $8,868,155(8.2)$ & $6,146,662(40.9)$ \\
\hline Short-term use & $6,337,179(4.2)$ & $5,136,073(4.7)$ & $1,201,106(19.0)$ \\
\hline MEQs Dispensed* & Millions (column \%) & Millions (column \%) & Millions (\% reduction) \\
\hline Total & $6122(100)$ & $4149(100)$ & $1973(32.2)$ \\
\hline Long-term use & $5651(92.3)$ & $3866(93.1)$ & $1785(31.6)$ \\
\hline Intermediate use & $352(5.7)$ & $191(4.6)$ & $161(45.8)$ \\
\hline Short-term use & $119(1.9)$ & $92(2.2)$ & $27(22.9)$ \\
\hline Supply Days per Fill & Mean (SD) & Mean (SD) & Mean (\% reduction) \\
\hline Total & $22.6(10.6)$ & $20.1(10.8)$ & $2.5(11.1)$ \\
\hline Long-term use & $28.9(3.2)$ & $28.4(2.8)$ & $0.5(1.7)$ \\
\hline Intermediate use & $21.4(11.3)$ & $18.2(10.0)$ & $3.2(15.0)$ \\
\hline Short-term use & $16.0(11.6)$ & $13.3(10.5)$ & $2.7(16.9)$ \\
\hline Daily Dose $^{\dagger}$ & Mean (SD) & Mean (SD) & Mean (\% reduction) \\
\hline Total & $35.5(54.7)$ & $33.7(40.1)$ & $1.8(5.1)$ \\
\hline Long-term use & $50.2(73.2)$ & $47.0(54.7)$ & $3.2(6.4)$ \\
\hline Intermediate use & $26.1(32.0)$ & $27.0(26.9)$ & $-0.9(-3.4)$ \\
\hline Short-term use & $23.7(30.5)$ & $24.7(22.1)$ & $-1.0(-4.2)$ \\
\hline
\end{tabular}

*Total morphine equivalents (MEQ) dispensed, in millions of milligrams ${ }^{10}$

TTime-weighted mean daily dose over the course of the year, expressed in milligrams of morphine equivalents per day ${ }^{10}$

discontinuing $(73,052-68,180=4872)$, new long-term use accounted for more than $90 \%(59,434 / 64,306)$ of the decreasing prevalence of long-term opioid use in the VHA.

The overall likelihood of transitioning to long-term opioid use decreased from $2.8 \%$ to $1.1 \%$ during the observation period, and decreased within all individual categories of prior opioid use (Table 2). Among patients with no use in the prior year, $1.1 \%$ of patients during 2010 and $0.4 \%$ of patients during 2015 developed subsequent long-term opioid use; $6.7 \%$ of short-term users during 2010 compared to $2.6 \%$ during 2015 , and $24.4 \%$ of intermediate users during 2010 compared to $14.1 \%$ during 2015 . These risks further demonstrated that prior opioid exposure was associated with subsequent risk for long-term opioid use in a stepwise manner. The relative risk of transitioning to long-term use during 2016 was 6.5 (95\% CI: $6.4,6.7$ ) among short-term users and 35.5 (95\% CI: 34.8, 36.3) among intermediate users, relative to patients with no opioid prescriptions filled during 2015.

\section{DISCUSSION}

On the surface, opioid prescribing trends followed similar trajectories in VHA and non-VHA settings, peaking around 2012 and subsequently declining. ${ }^{7,9}$ We observed a decrease of $16 \%$ in overall opioid prevalence between 2012 and 2015 , compared to $13 \%$ reported in non-VHA settings. ${ }^{7}$ Importantly, our analysis provided a more finely tuned examination of VHA trends, revealing that the prevalence of short-term opioid prescribing decreased by approximately $7 \%$, while the prevalence of long-term and intermediate-term opioid prescribing decreased by approximately one-third from 2012 through 2016. These findings stand in some contrast to nonVHA settings. The average supply days per prescription decreased from 2010 through 2016 in the VHA, reflecting an underlying decrease in long-term opioid use. However, in a study of U.S. retail prescription data, this parameter increased through 2015 , driven primarily by a shift toward more 30-day prescriptions, which may indicate a parallel rise in long-term opioid use in the community setting. ${ }^{7}$ More direct evidence comes from National Health and Nutrition Examination Survey data, which demonstrated a decrease in short-term opioid use from 2012 to 2014 , but an increase in long-term use during this period. ${ }^{12}$

The relative stability of short-term opioid prescribing rates within the VHA is consistent with observations from prior time periods. From 2004 to 2012, incident opioid prescribing increased by only $11 \%$, where newly prescribed opioids generally reflect short-term use for acute pain. ${ }^{8}$ The fact that shortterm opioid prevalence decreased by only $7 \%$ from 2012 to 2016 should not be surprising given that incidence rates had increased by a similarly small degree during the prior decade. Short-term opioid therapy has a clear therapeutic role in the management of acute pain. ${ }^{13}$ While the optimal rate of shortterm opioid prescribing in the VHA is unknown, the dose and number of supply days dispensed should be minimized when prescribed, and non-opioid alternatives should be employed when clinically appropriate. ${ }^{13-15}$ Minimizing opioid prescribing for acute pain is important not only for reducing risk among patients to whom they are dispensed, but also in reducing the overall opioid supply distributed and available 


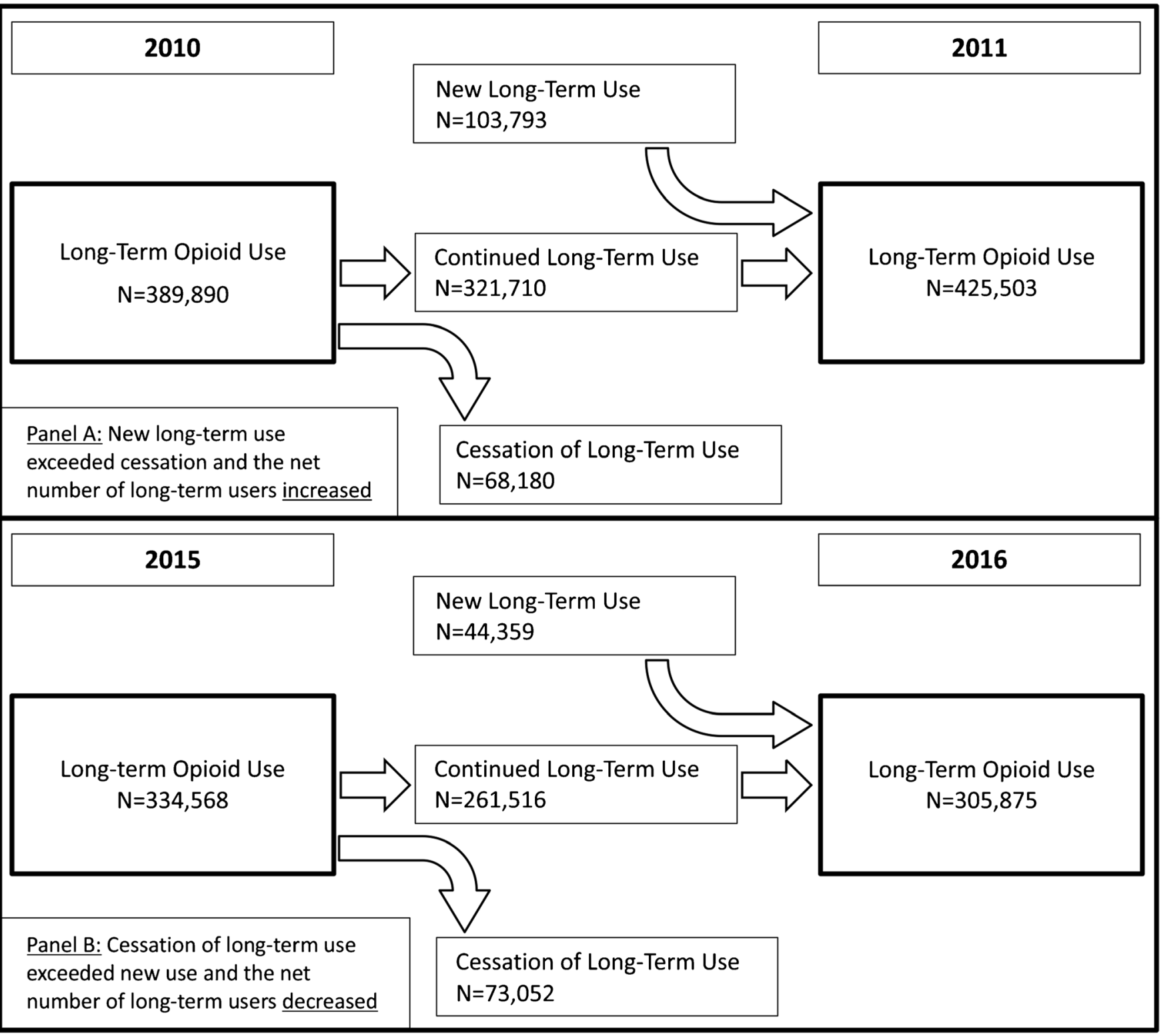

Figure 2 Shift toward decreasing chronic opioid use of as a function of the input of new chronic use and output of cessation of chronic use.

to the public at large. In keeping with this concept, mean supply per prescription decreased by 2.7 days among shortterm opioid users in the VHA from 2010 to 2016, and decreased by 2.5 days among all users. In sharp contrast, data from the non-VHA retail setting demonstrated an increase in average days' supply per prescription of $2-3$ days between 2010 and 2015 . $^{7}$

One striking observation was the decrease in the prevalence of long-term opioid therapy from a peak of $9.5 \%$ in 2012 to $6.2 \%$ in 2016. This trend is particularly notable given that rates of long-term opioid prescribing outside the VHA system appeared stable, if not potentially increasing during this period. ${ }^{7}$ While minimizing short-term opioid exposure is important, the clear majority of opioid harms occur among long-term users, and these individuals accounted for $80-90 \%$ of VHA opioid prescribing. Similarly, opioid prescribing increases in the VHA prior to 2012 were largely driven by long-term use, where opioid prevalence nearly doubled from 2004 , and median calendar days of exposure increased from 87 to 147 days. $^{8}$ Therefore, declining rates of long-term opioid use in the VHA seem to be a favorable reversal of prior prescribing practices, particularly in light of recent CDC guidelines calling for more judicious use of long-term opioid therapy in the treatment of chronic pain. $^{5}$

More detailed examination revealed that recent decreases in long-term opioid prescribing did not occur through discontinuation among existing users, but principally through reductions in the number of new long-term users. It is well documented and widely understood among practicing clinicians that long-term opioid therapy is infrequently discontinued once initiated. ${ }^{16}$ Therefore, it is little surprise that the path of least resistance in curtailing opioid prescribing would be to 
Table 2 Likelihood of Transitioning to Long-Term Use by Duration of Use Category in the Prior Year

\begin{tabular}{|c|c|c|c|c|}
\hline \multirow{3}{*}{$\begin{array}{l}\text { Opioid Use } \\
\text { in First Year }\end{array}$} & \multicolumn{4}{|c|}{ Likelihood of Long-Term Opioid Use in Second Year } \\
\hline & \multicolumn{2}{|l|}{2010 to 2011} & \multicolumn{2}{|l|}{2015 to 2016} \\
\hline & $\mathrm{n} / \mathbf{N}^{*}(\%)$ & RR $(95 \%$ CI $)$ & $\mathrm{n} / \mathbf{N}^{*}(\%)$ & RR $(95 \%$ CI $)$ \\
\hline All groups & $103,793 / 3,735,564(2.8)$ & & 44,359 / 4,037,387 (1.1) & \\
\hline None & $36,715 / 3,240,942(1.1)$ & 1.0 [Reference] & $14,106 / 3,557,564(0.4)$ & 1.0 [Reference] \\
\hline Short-term & $20,340 / 302,969(6.7)$ & $5.9(5.8,6.0)$ & 8439 / 324,993 (2.6) & $6.5(6.4,6.7)$ \\
\hline Intermediate & 46,738 / 191,653 (24.4) & $21.5(21.3,21.8)$ & $21,814 / 154,830(14.1)$ & $35.5(34.8,36.3)$ \\
\hline
\end{tabular}

*Denominators included all patients that were VHA pharmacy users during both years of analysis and were not existing long-term users in the earlier of the two years

$R R=$ relative risk; $C I=$ confidence interval

focus first on minimizing new long-term use, presumably by emphasizing the role of alternative pharmacologic and nonpharmacologic therapies. Overall, the likelihood of new longterm opioid use decreased from $2.8 \%$ in 2011 to $1.1 \%$ in 2016, and this trend was observed across all classifications of prior opioid use, including non-users, short-term users, and intermediate users.

Though determining the causal mechanisms underlying observed trends was beyond the scope of our study, the VHA has undertaken several initiatives that may explain our findings. ${ }^{9}$ The 2010 Department of Veterans Affairs and Department of Defense (VA/DoD) Clinical Practice Guideline for Management of Opioid Therapy for Chronic Pain ${ }^{17}$ emphasized a goal-directed approach to trialing opioid therapy in chronic pain, careful patient selection and monitoring, and weaning if treatment goals are not reached. These guidelines have since been updated with a recommendation against initiation of long-term opioid therapy for chronic pain. ${ }^{18}$ Further, VHA has expanded access to complementary treatments and multimodal therapy for chronic pain. In 2009 , two VHA directives were issued addressing the availability of chiropractic care ${ }^{19}$ and implementation of stepped care, ${ }^{20}$ emphasizing a biopsychosocial understanding of chronic pain. Increased provision of alternatives to opioid therapy may have contributed to decreased opioid use. Lastly, the VA Opioid Safety Initiative (OSI) implemented nationally in 2013 focused on specific clinical safety targets such as high-dose use and concurrent benzodiazepine prescribing, as well as monitoring via urine drug screens and state prescription drug monitoring databases. ${ }^{21}$ Although the OSI was primarily targeted at existing long-term opioid users, it is possible the initiative helped accelerate a change in prescribing culture that has multiple influences, including media coverage of opioids and opioid-related deaths, and non-VA policies or practice guidelines.

The primary limitation to our study was the inability to observe opioid prescriptions filled by non-VA pharmacies. First, our observation that $80-90 \%$ of opioid prescribing in the VHA was accounted for by long-term use is true from the system perspective, but may be overestimated from the patient perspective. Patients suffering from acute pain may be more likely than those with chronic pain to seek care outside the
VHA because it is closer to home or otherwise easier to access on an acute basis. Moreover, patients with acute pain seen at VHA community-based outpatient clinics, many without a dispensing pharmacy on-site, may preferentially fill opioid prescriptions at a local non-VA pharmacy. Thus, opioid prescribing data within the VHA is likely skewed toward long-term use relative to opioid prescriptions dispensed to veterans across all healthcare systems and settings. In addition, under-identification of opioid prescriptions from non-VHA sources would result in misclassification of patients into lower exposure categories. For example, patients may have been classified as intermediate users in our analysis, but would be classified as long-term users if all opioid prescriptions were observable. An additional limitation is that this analysis does not consider potential changes in rates of underlying pain diagnoses or other patient characteristics over time that might be associated with opioid prescribing. However, it seems unlikely that there has been a precipitous drop in the prevalence of chronic pain or other such characteristics over the past few years that would account for the magnitude of change observed in long-term opioid prescribing.

\section{CONCLUSION}

Opioid prescribing trends followed similar trajectories in VHA and non-VHA settings, peaking around 2012 and subsequently declining. More detailed analysis revealed that changes in long-term opioid prescribing accounted for most of this decline in the VHA, whereas the primary driver in nonVHA retail pharmacy data seemed to be decreases in shortterm use. ${ }^{7}$ This apparent discrepancy suggests that recent VHA initiatives to curb the opioid epidemic may be having a direct and specific benefit in minimizing potential harms from exposure to long-term opioid therapy-particularly by preventing patients from initiating long-term use. Future work toward understanding precisely which initiatives have most positively impacted opioid prescribing would be needed to maintain effective approaches within the VHA, and as importantly, would offer valuable lessons that may be generalizable to other healthcare systems, providers, and patients. 
Acknowledgements: This research was supported by the Iowa City VA Health Care System, Department of Pharmacy Services. Additional support was provided by the Health Services Research and Development Service, Department of Veterans Affairs (Drs. Lund and Mosher, CIN 13-412). None of these sponsors had any role in the study design, methods, analyses, or interpretation, or in the preparation of the manuscript and decision to submit it for publication. The views expressed in this article are those of the authors and do not necessarily reflect the position or policy of the Department of Veterans Affairs.

Corresponding Author: Brian C. Lund, PharmD; Center for Comprehensive Access and Delivery Research and Evaluation (CADRE) Iowa City VA Healthcare System, Iowa City, IA, USA (e-mail: brian. lund@va.gov).

\section{Compliance with Ethical Standards:}

Conflict of Interest: The authors declare that they have no conflict of interest.

\section{REFERENCES}

1. Prescription Drug Monitoring Program Training and Technical Assistance Center. Available at: http://www.pdmpassist.org. Accessed December 12, 2017.

2. Wen H, Schackman BR, Aden B, Bao Y. States with prescription drug monitoring mandates saw a reduction in opioids prescribed to Medicaid enrollees. Health Aff (Millwood). 2017;36:733-41.

3. Drug Enforcement Administration, Department of Justice. Schedules of controlled substances: rescheduling of hydrocodone combination products from schedule III to schedule II. Final rule. Fed Regist. 2014;79(163):49661-82.

4. Huff, C. States aim to limit opioid prescriptions. ACP Internist. 2016. Available at: https://acpinternist.org/archives/2016/10/laws.htm. Accessed December 12, 2017.

5. Dowell D, Haegerich TM, Chou R. CDC guideline for prescribing opioids for chronic pain - United States, 2016. MMWR Recomm Rep. 2016;65:1-49.

6. S. 524 Comprehensive Addiction and Recovery Act of 2016. Public Law 114-198, 114th Congress. July 22, 2016. https://www.congress.gov/ bill/114th-congress/senate-bill/524/text. Accessed 16 Jan 2018

7. Guy GP, Zhang $\mathbf{K}$, Bohm MK, et al. Vital signs: changes in opioid prescribing in the United States, 2006-2015. MMWR Morb Mortal Wkly Rep. 2017;66:697-704.

8. Mosher HJ, Krebs EE, Carrel M, Kaboli PJ, Vander Weg MW, Lund BC. Trends in prevalent and incident opioid receipt: an observational study in
Veterans Health Administration 2004-2012. J Gen Intern Med. 2015;30:597-604.

9. Gellad WF, Good CB, Shulkin DJ. Addressing the opioid epidemic in the United States: lessons from the Department of Veterans Affairs. JAMA Intern Med. 2017;177:611-2.

10. Centers for Medicare \& Medicaid Services. Available at: https://www.cms. gov/Medicare/Prescription-Drug-Coverage/PrescriptionDrugCovContra/ Downloads/Opioid-Morphine-EQ-Conversion-Factors-March-2015.pdf. Accessed December 12, 2017.

11. Mosher HJ, Richardson KK, Lund BC. The 1-year treatment course of new opioid recipients in Veterans Health Administration. Pain Med. 2016; 17:1282-91.

12. Mojtabai R. National trends in long-term use of prescription opioids. Pharmacoepidemiol Drug Saf. 2017; doi: https://doi.org/10.1002/pds. 4278.

13. Chou R, Gordon DB, de Leon-Casasola OA. Management of postoperative pain: a clinical practice guideline from the American Pain Society, the American Society of Regional Anesthesia and Pain Medicine, and the American Society of Anesthesiologists' Committee on Regional Anesthesia, Executive Committee, and Administrative Council. J Pain. 2016;17:131-57.

14. Mylonas KS, Reinhorn M, Ott LR, Westfal ML, Masiakos PT. Patientreported opioid analgesic requirements after elective inguinal hernia repair: a call for procedure-specific opioid-administration strategies. Surgery. 2017;162:1095-100.

15. Brummett CM, Waljee JF, Goesling J, et al. New persistent opioid use after minor and major surgical procedures in US adults. JAMA Surg. 2017; 152:e170504.

16. Vanderlip ER, Sullivan MD, Edlund MJ, et al. National study of discontinuation of long-term opioid therapy among veterans. Pain. 2014;155:2673-9.

17. VA/DoD Clinical Practice Guideline. Management of opioid therapy for chronic pain. Available at: https://www.va.gov/painmanagement/docs/ cpg_opioidtherapy_fulltext.pdf. Accessed December 12, 2017.

18. VA/DOD Clinical Practice Guideline. Opioid therapy for chronic pain. Available at: https://www.healthquality.va.gov/guidelines/Pain/cot/ VADoDOTCPG022717.pdf. Accessed December 12, 2017.

19. Department of Veterans Affairs. VHA Directive 2009-059: Chiropractic Care. Available at: https://www.va.gov/vhapublications/ViewPublication. asp?pub_ID=2117. Accessed December 12, 2017.

20. Department of Veterans Affairs. VHA Directive 2009-053: Pain management. Available at: https://www.va.gov/painmanagement/docs/ vha09paindirective.pdf. Accessed December 12, 2017.

21. Lin LA, Bohnert ASB, Kerns RD, Clay MA, Ganoczy D, Ilgen MA. Impact of the Opioid Safety Initiative on opioid-related prescribing in veterans. Pain. 2017;158:833-9. 